\title{
Factores nutricionales asociados al crecimiento de recién nacidos prematuros menores de 1,500 gramos en la Unidad de Cuidado Intensivo Neonatal del Hospital de San José, Bogotá DC, Colombia
}

\author{
Bladimir Marín-Montoya ${ }^{1 *}$, Marilyn Zea ${ }^{1}$, Agatha León ${ }^{1}$, Brenda Núñez ${ }^{2}$, Cristhian Rodríguez ${ }^{2}$, \\ Andrés Meza ${ }^{2}$ y María Zúñiga ${ }^{3}$ \\ ${ }^{1}$ Departamento de Pediatría; ${ }^{2}$ Servicio Neonatología; ${ }^{3}$ Servicio de Especialización Epidemiología Clínica. Fundación Universitaria de Ciencias de
} la Salud, Hospital de San José de Bogotá, Bogotá, Colombia

\section{Resumen}

Objetivo: Analizar y describir los factores nutricionales asociados al crecimiento de los recién nacidos prematuros (RNPT) con muy bajo peso al nacer $(<1,500 \mathrm{~g})$, hospitalizados en una unidad de cuidados intensivos neonatales. Materiales y métodos: Estudio de cohorte, observacional, analítico y retrospectivo en recién nacidos con muy bajo peso al nacer mediante determinación de sujetos expuestos (peso bajo para la edad gestacional [PBEG]) y sujetos no expuestos (peso adecuado para la edad gestacional [PAEG]). Se analizaron datos antropométricos, nutricionales, demográficos, factores maternos, fetales y placentarios. Los resultados se presentan en frecuencias absolutas y relativas. Se realizó un análisis de regresión logística multivariado para establecer el carácter predictivo de variables y bivariado para determinar el riesgo de no lograr una condición nutricional adecuada. Resultados: Ingresaron 141 recién nacidos que cumplían los criterios de inclusión. La población se agrupó según la edad gestacional (EG): menores de 28 semanas, de 28-32 semanas y mayores de 32 semanas. El 57.45\% correspondía al grupo de 28-32 semanas de EG. Respecto al peso al nacer, la mayoría de los RNPT del estudio tenían un peso > 1,250 $\mathrm{g}(n=91,64.54 \%)$. Variables como la estancia hospitalaria, OR 1.6 (1.02-1.11), y la EG al nacer, 1 (0.73-1.99), son predictivas con respecto al estado nutricional de los prematuros con PBEG. Conclusión: Los prematuros con PBEG y mayor estancia hospitalaria presentan un mayor riesgo de no alcanzar la condición nutricional ideal que aquellos con PAEG.

Palabras clave: Prematuro. Bajo peso al nacer. Pequeño para edad gestacional. Nutrición. Velocidad de crecimiento.

\section{Nutritional factors associated with the growth of premature newborns under 1500 grams in the Neonatal Intensive Care Unit of San José Hospital, Bogotá DC, Colombia}

\section{Abstract}

Objective: To analyze and describe the nutritional factors associated with the growth of premature infants (PWNN) with very low birth weight $(<1,500 \mathrm{~g})$, hospitalized in a neonatal intensive care unit. Materials and methods: Cohort, observational, analytical and retrospective study in infants with very low birth weight by determination of exposed subjects (low weight for

Correspondencia:

*Bladimir Marín-Montoya E-mail: bladi2280@gmail.com
Disponible en internet: 18-12-2019 Perinatol Reprod Hum. 2019;33:72-79 www.perinatologia.mx 0187-5337/C 2019. Instituto Nacional de Perinatología Isidro Espinosa de los Reyes. Publicado por Permanyer. Este es un artículo open access bajo la licencia CC BY-NC-ND (http://creativecommons.org/licenses/by-nc-nd/4.0/). 
gestational age [PBEG]) and unexposed subjects (adequate weight for gestational age [PAEG]). We analyzed anthropometric, nutritional, demographic, maternal, fetal and placental factors, the results are presented in absolute and relative frequencies. A multivariate logistic regression analysis was performed to establish the predictive nature of variables and bivariate to determine the risk of not achieving adequate nutritional condition. Results: 141 RNs that met the inclusion criteria were included. The population was grouped according to gestational age (GD), under 28 weeks, 28-32 weeks, and over 32 weeks. The $57.45 \%$ corresponded to the group of 28-32 weeks EG, with respect to the weight at birth, the majority of the RNPT of the study were $>1,250 \mathrm{~g}(n=91,64.54 \%)$. Variables such as hospital stay $1.6(1.02-1.11)$, and $E G$ at birth 1 (0.73-1.99) are predictive of the nutritional status of preterm infants with PBEG. Conclusion: Premature with PBEG and longer hospital stay present a greater risk of not reaching the ideal nutritional condition than those of PAEG.

Key words: Preterm. Low birth weight. Small for gestational age. Nutrition. Growth rate.

\section{Introducción}

En la década de 1960 surgen las unidades de cuidados intensivos neonatales (UCIN), con resultados favorecedores en la supervivencia de los recién nacidos, especialmente los muy prematuros $(<1500 \mathrm{~g})$, y con ello el aumento en el conocimiento de esta población y sus complicaciones futuras, entre estas, el crecimiento y el desarrollo neurológico'.

La Organización Mundial de la Salud (OMS) define como prematuro al que nace antes de las 37 semanas de edad gestacional (EG). El prematuro, a su vez, se subdivide según su EG en: prematuro extremo (menores de 28 semanas), muy prematuro (menores 32 semanas) y prematuro moderado o tardío (entre la 32-37 semanas). También se clasifica según el peso en: peso bajo al nacer (menos de 2,500 g), independientemente de la EG, peso muy bajo al nacer (menos de 1,500 g) y peso extremadamente bajo al nacer (menos de $1,000 \mathrm{~g})^{2}$.

Por otra parte, es importante diferenciar el prematuro con peso adecuado para la edad gestacional (PAEG) del prematuro con peso bajo para la edad gestacional (PBEG). Este último se define cuando su peso y/o longitud se encuentran dos o más desviaciones estándar (DE) por debajo de la media establecida para su población de referencia, su sexo y su EG ${ }^{3,4}$. Los pacientes con PBEG están generalmente asociados con la restricción del crecimiento intrauterino (RCIU), causada por un suministro inadecuado de oxígeno y nutrientes, atribuibles en la mayoría de los casos a insuficiencia placentaria 5 .

Se estima que anualmente nacen en el mundo 14.9 millones (rango de 12.3 a 18.1 millones) de niños antes de las 37 semanas de gestación, lo que representa un $11.1 \%$ de todos los recién nacidos vivos ${ }^{2}$. El nacimiento prematuro es una de las causas de mortalidad en niños menores de 5 años, y se considera la segunda causa de muerte después de la neumonía en esta población, y es responsable del $35 \%$ de las muertes neonatales anuales $^{6}$. Por ello, se han creado estrategias de manejo para esta población, entre las más importantes se hallan las nutricionales, con el objetivo de reducir su mortalidad y morbilidad.

Los recién nacidos presentan una pérdida de peso en la primera semana de vida. Esto se debe a la pérdida de agua corporal, que es mayor cuanto menor es la EG? Una vez recuperado el peso al nacer, se observan bajas velocidades de crecimiento durante las primeras semanas de vida. La administración temprana de nutrientes es un determinante importante del crecimiento posnatal. Se ha demostrado que las prácticas nutricionales en los primeros 7 días de nacido se asocian con la velocidad de crecimiento entre el día 7 y el día $28^{8}$.

Los prematuros de muy bajo peso al nacer presentan un aumento de los requerimientos nutricionales, dado a que su propia prematuridad los predispone a condiciones clínicas de mayor estrés, lo que aumenta aún más su estado catabólico, que asociado a una inadecuada nutrición, enteral o parenteral, contribuye a una alteración en el crecimiento de los recién nacidos prematuros hospitalizados ${ }^{9}$. Los periodos de crecimiento lento que ocurren durante los episodios de infecciones o intolerancia alimentaria necesitan ser compensados con un crecimiento acelerado en otro periodo ${ }^{10}$. Un crecimiento deficiente en las etapas tempranas de la vida, en donde pasan por un periodo de hiperplasia e hipertrofia celular, puede tener efectos perjudiciales permanentes, una alteración en el crecimiento en la etapa neonatal puede persistir en la edad adulta ${ }^{1}$.

Una vez que el prematuro recupera el peso al nacer, el crecimiento se dirige a los objetivos estimados para el crecimiento intrauterino. Esto se describe a partir de estudios históricos de cohorte de niños nacidos vivos con diferentes edades gestacionales. Un crecimiento esperado para prematuros es una ganancia de peso de 15 a $18 \mathrm{~g} / \mathrm{kg} / \mathrm{día}$, de talla de $1 \mathrm{~cm} / \mathrm{semana}$ y de perímetro cefálico de $0.7 \mathrm{~cm} / \mathrm{semana}^{11}$. 
Por esta razón es de suma importancia el cuidado nutricional y el seguimiento antropométrico de los recién nacidos, especialmente los menores de 1,500 gramos en las UCIN, y uno de los objetivos de los profesionales de las UCIN es proporcionar una nutrición adecuada para lograr una velocidad de crecimiento similar a la velocidad de crecimiento intrauterina. El siguiente estudio tiene como objetivo analizar y describir los factores nutricionales asociados al crecimiento de los recién nacidos prematuros de menos de 1,500 gramos, hospitalizados en la UCIN del Hospital de San José de Bogotá DC, por un periodo de tiempo de 4 años.

\section{Materiales y métodos}

Se efectuó un estudio de cohorte, observacional, analítico y retrospectivo a partir de datos primarios (historia clínica) de la UCIN del Hospital de San José de Bogotá. Se seleccionaron pacientes prematuros con muy bajo peso al nacer, nacidos en el hospital e internados en la UCIN entre los años 2013 y 2016. Se excluyeron del estudio aquellos con malformaciones congénitas mayores, remitidos, fallecidos e historias clínicas incompletas. La muestra se dividió en dos grupos: prematuros con PBEG, que correspondían a los expuestos, y prematuros con PAEG, que correspondían a los no expuestos.

\section{Métodos estadísticos}

Los datos fueron recolectados por el grupo de investigadores y se registraron en un cuestionario multicomponente, dividido en tres categorías de variables (maternas, fetales y nutricionales). Se creó una matriz de datos apilados en Microsoft Excel 2014, y en ella se ingresaron las medidas antropométricas registradas al ingreso a la UCIN. La talla y el perímetro cefálico se recolectaron semanalmente, y se efectuó un promedio semanal del peso, teniendo en cuenta el registro diario reportado por enfermería. Se registró el método nutricional utilizado desde el momento del nacimiento (enteral y parenteral) y se efectuó, de igual forma, un promedio semanal durante la estancia hospitalaria. La variable de desenlace del estudio se denominó «velocidad de crecimiento", la cual se definió como el puntaje $z$ del peso al nacer menos el puntaje $z$ del peso al egreso. Se definió como desnutrición extrauterina tener una velocidad de crecimiento por debajo de -1 . Los datos de la matriz se exportaron al programa Stata $14^{\circledR}$ para la consecución de los resultados. Los datos categóricos se presentan en unidades de frecuencia y porcentajes.
La distribución de los datos continuos de interés se expresa en medias y desviación estándar según la prueba de Kolmogorov-Smirnov. Se efectuó un análisis bivariado y una regresión logística multivariada para determinar la asociación entre nutrición extrauterina alcanzada y los factores asociados. Para el modelo se utilizó una técnica forward con variables significativas en el análisis bivariado o de alta relevancia clínica, y se revisaron los supuestos del modelo mediante prueba de homogeneidad y curvas Receiver Operating Characteristic (ROC). Para determinar el efecto de la nutrición, se realizó un análisis de varianza de medidas repetidas. Se utilizó significancia estadística, intervalos de confianza del $95 \%$ y valores de $p$ menores 0.05 . Esta investigación fue aprobada por el Comité de ética e investigación de la Fundación Universitaria de Ciencias de la Salud, y se clasifica como una investigación sin riesgo, por lo cual no necesitó de consentimiento informado.

\section{Resultados}

Durante el periodo de estudio ingresaron a la cohorte 141 recién nacidos que cumplían los criterios de inclusión. En cuanto a las variables sociodemográficas, la edad de las madres presentó una media de $29.3 \pm 6.9$ años. El $70.3 \%(n=99)$ fueron multíparas, el $73.05 \%$ $(n=103)$ presentaron gestación única, y los nacimientos por cesárea se presentaron en un $97.16 \%(n=137)$ (Tabla 1).

Los recién nacidos presentaron una estancia promedio de 39.3 días. El $53.9 \%$ de la muestra correspondió al género femenino. La población en estudio se agrupó en tres categorías según la EG, menores de 28 semanas, entre 28 semanas y menos de 32 semanas, y mayores de 32 semanas, de los cuales el grupo entre las 28 semanas y menos de 32 semanas presentaron la mayor frecuencia, con un $57.45 \%$. Con respecto al peso al nacer, se encontró que la mayoría de los recién nacidos prematuros del estudio presentaron un peso mayor a 1,250 gramos (64.54\%) (Tabla 2). Presentaron un valor $z$ de peso al nacer menor -2 DE (RCIU confirmada) el $13.48 \%(n=19)$.

El $93 \%$ de los recién nacidos $(n=131)$ presentaron síndrome de dificultad respiratoria, y la causa más frecuente fue el déficit de surfactante (68.6\%) (Tabla 3 ).

De los factores de riesgo fetales, el más frecuentemente mencionado fue la restricción de crecimiento intrauterino, con un $29.73 \%(n=42)$, seguido por el embarazo gemelar, con un $17.73 \%(n=25)$. En cuanto a los factores de riesgo placentario, se encontró que las anomalías placentarias, al igual que las de cordón, 
Tabla 1. Características sociodemográficas de las madres de los recién nacidos

\begin{tabular}{|l|c|c|}
\hline Ítem & Media & DE \\
\hline Edad & 29.3 & 6.9 \\
\hline Estado civil & Número & Porcentaje \\
Unión libre & 54 & 38.30 \\
Sin dato & 45 & 31.91 \\
Casadas & 20 & 14.18 \\
Solteras & 12 & 8.51 \\
\hline Total & 131 & 100 \\
\hline Paridad & Número & Porcentaje \\
Multípara & 99 & 70.21 \\
Primigestante & 42 & 29.79 \\
\hline Total & 141 & 100 \\
Gestación & Número & Porcentaje \\
Única & 103 & 73.05 \\
Múltiple & 38 & 26.95 \\
\hline Total & 141 & 100 \\
Tipo de nacimiento & Numero & Porcentaje \\
Cesárea & 137 & 97.16 \\
Vaginal & 4 & 2.84 \\
Total & 141 & 100 \\
& &
\end{tabular}

Fuente: Elaborada por los autores a partir de la base de datos.

Tabla 2. Distribución de la edad gestacional y peso al nacer de los recién nacidos

\begin{tabular}{|l|c|c|}
\hline Ítem & Número & Porcentaje \\
\hline $\begin{array}{l}\text { Edad gestacional } \\
\text { Menores de } 28 \text { semanas }\end{array}$ & 6 & 4.26 \\
$\begin{array}{l}\text { 28 semanas a menos de 32 } \\
\text { semanas }\end{array}$ & 81 & 57.45 \\
De 32 semanas o más & 54 & 38.3 \\
\hline Total & 141 & 100 \\
\hline Peso al nacer & & \\
1,250 g o más & 91 & 64.54 \\
$\quad$ Entre 1,000 y 1,250 g & 40 & 28.37 \\
Menos de 1,000 g & 10 & 7.09 \\
\hline Total & 141 & 100 \\
\hline
\end{tabular}

Fuente: Elaborada por los autores a partir de la base de datos.

fueron escasas (Tabla 4). El $68.79 \%$ de los prematuros recibió soporte con nutrición parenteral a lo largo de su estancia.

Durante la construcción del modelo de regresión logística para establecer la repercusión que tienen las variables de exposición determinadas en el estudio con el adecuado crecimiento de los recién
Tabla 3. Distribución de causas de síndrome de dificultad respiratoria de los recién nacidos

\begin{tabular}{|l|c|c|}
\hline Ítem & Número & Porcentaje \\
\hline Déficit de surfactante & 97 & 68.6 \\
\hline Neumonía in utero & 16 & 11.4 \\
\hline Taquipnea transitoria del recién nacido & 14 & 9.8 \\
Sin causa determinada & 3 & 2.4 \\
\hline Fuga aérea & 1 & 0.8 \\
\hline Aspiración de meconio & 0 & 0.2 \\
\hline Total & 131 & 93
\end{tabular}

Fuente: Elaborada por los autores a partir de la base de datos.

Tabla 4. Distribución de los factores de riesgo fetales y placentarios

\begin{tabular}{|l|c|c|}
\hline Riesgos fetales & Número & Porcentaje \\
\hline Ninguno & 70 & 49.6 \\
$\begin{array}{l}\text { Restricción del crecimiento } \\
\text { intrauterino }\end{array}$ & 42 & 29.73 \\
\hline Gemelar & 25 & 17.73 \\
\hline Anomalías cromosómicas & 2 & 1.4 \\
\hline Malformaciones cardíacas & 1 & 0.77 \\
\hline Malformaciones renales & 1 & 0.77 \\
\hline Total & 141 & 100 \\
\hline Riesgos placentarios & Número & Porcentaje \\
\hline Ninguno & 136 & 96.45 \\
\hline Anomalías placentarias & 3 & 2.13 \\
\hline Anomalías del cordón & 2 & 1.42 \\
\hline Total & 141 & 100 \\
\hline Fuente: Elaborada por los autores a partir de la base de datos. & & \\
\hline
\end{tabular}

nacidos con menos de 1,500 gramos hospitalizados en la unidad de cuidado intensivo neonatal, encontramos que las variables independientes predictoras que resultaron significativas en el análisis bivariado son: la estancia hospitalaria, 1.6 (1.02-1.11); RCIU confirmada, 1.6 (1.11-2.33); edad de inicio de la nutrición enteral, 1.0 (1.00-1.10); uso o no de nutrición parenteral, 2.0 (0.98-4.68), y EG al nacer, 1 (0.731.99) (Tabla 5).

Al realizar el análisis de varianza de medidas repetidas con dos factores comparando pacientes con y sin 
Perinatol Reprod Hum. 2019;33

Tabla 5. Odds ratio de las variables intervinientes

\begin{tabular}{|l|c|c|}
\hline Variables & Delta Z1 OR (IC 95\%) & Delta Z2 OR (IC 95\%) \\
\hline Estancia & $1.0(1.018-1.080)$ & $1.6(1.02-1.11)$ \\
\hline Peso al nacer & $1.6(0.648-0.929)$ & $0.9(0.99-0.99)$ \\
\hline Restricción del crecimiento intrauterino & $0.2(0.102-0.813)$ & $1.6(1.11-2.33)$ \\
\hline Edad de inicio nutrición enteral & $1.5(1.097-2.124)$ & $1.0(1.00-1.10)$ \\
\hline Nutrición parenteral (sí o no) & $3.0(1.472-6.443)$ & $2.0(0.98-468)$ \\
\hline Edad gestacional al nacer & $0.71(0.648-0.929)$ & $1(0.73-1.99)$
\end{tabular}

Fuente: Elaborada por los autores a partir de la base de datos.

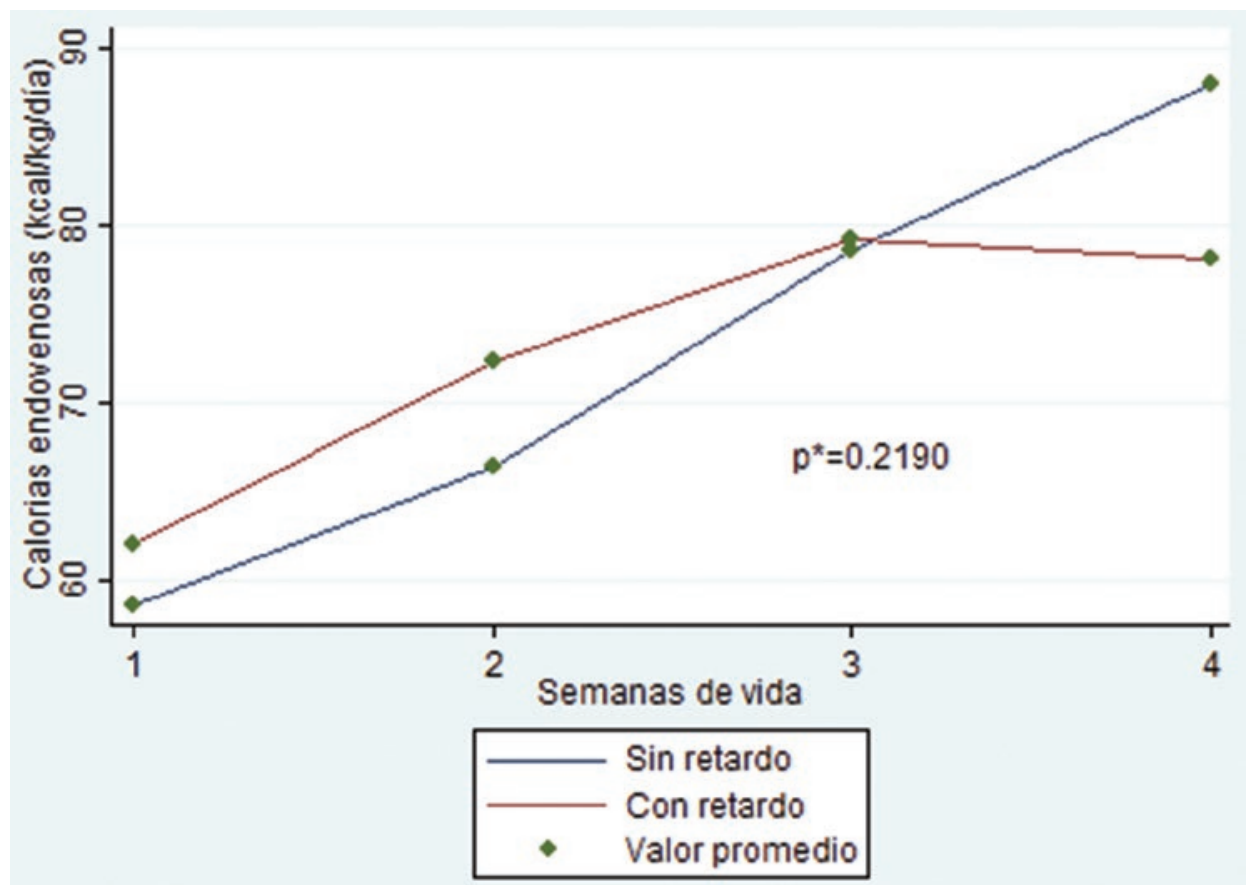

Figura 1. Comparación de promedio de kilocalorías recibidas por kilogramo de peso por vía endovenosa por día en el grupo de pacientes con y sin restricción del crecimiento intrauterino. Comparación por Anova de medidas repetidas de dos factores entre pacientes con y sin restricción extrauterina examinando el promedio de kilocalorías recibidas por kilogramo de peso por vía endovenosa por día. No se encontró una diferencia significativa entre los dos grupos $(p>0.05)$.

un adecuado crecimiento extrauterino, se observó que no hay diferencias estadísticamente significativas entre el promedio semanal de kilocalorías (kcal) endovenosas $(p=0.219)$ (Fig. 1) o kcal totales recibidas por kilogramo de peso por día entre los grupos $(p=0.11)$ (Fig. 2), pero sí en cuanto al volumen ( $\mathrm{ml} / \mathrm{kg} / \mathrm{día})$ de nutrición enteral por semanas recibido, donde se observó un mayor aporte por semana en el grupo sin restricción ( $p=0.014)$ (Fig. 3).
Se corrió un modelo de regresión logística multivariado con las variables significativas del análisis bivariado que ingresaron al modelo, encontrando que la estancia y la EG al nacer se constituyen en predictores, es decir, que a menor EG y mayor tiempo de estancia en la unidad de cuidado intensivo neonatal, la probabilidad de crecimiento en los recién nacidos prematuros menores a 1,500 gramos al nacer disminuye. 


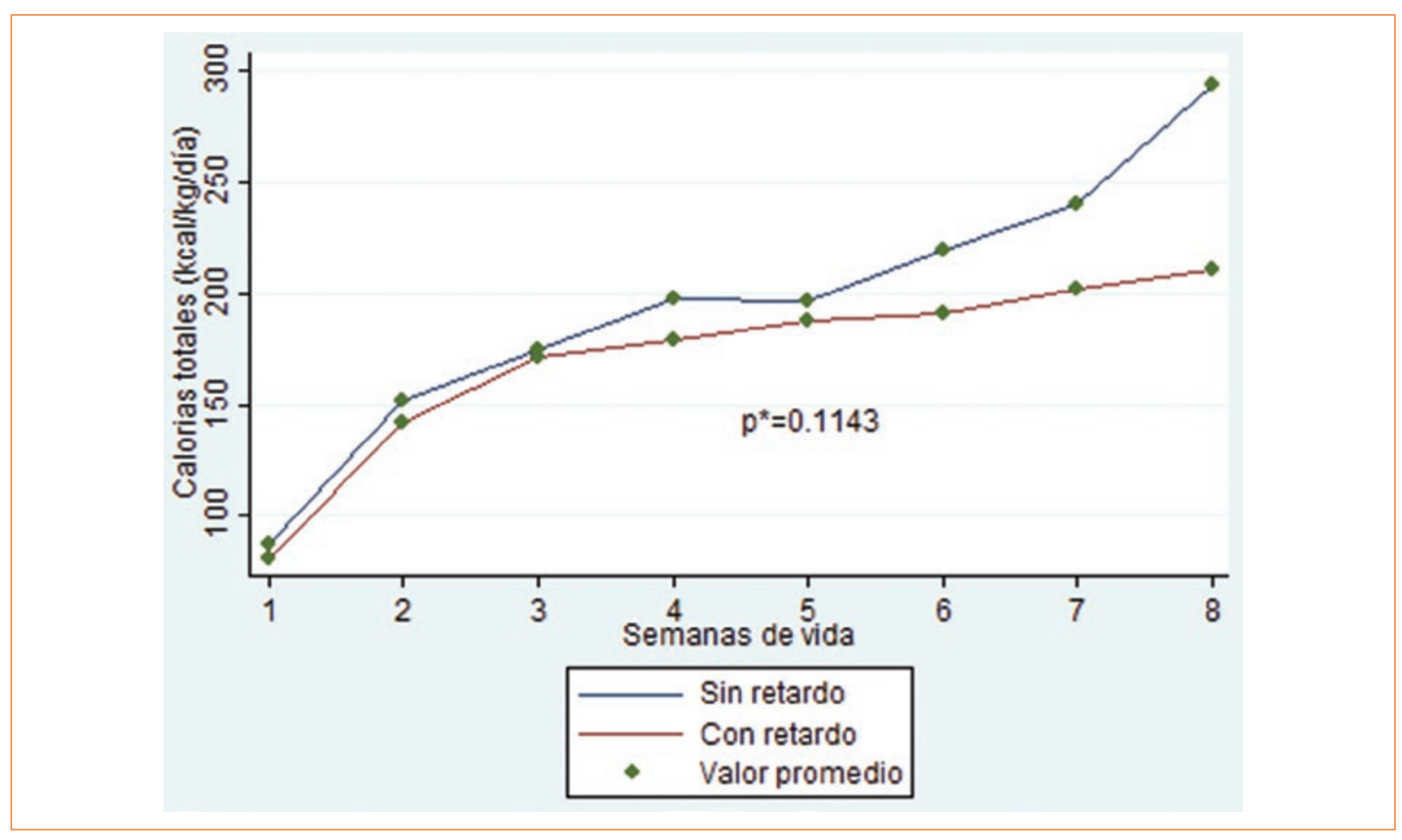

Figura 2. Comparación del promedio de kilocalorías totales en el grupo de pacientes con y sin restricción del crecimiento intrauterino. Comparación por Anova de medidas repetidas de dos factores entre pacientes con y sin restricción extrauterina examinando el promedio de kilocalorías totales recibidas. No se encontró una diferencia significativa entre los dos grupos $(p>0.05)$.

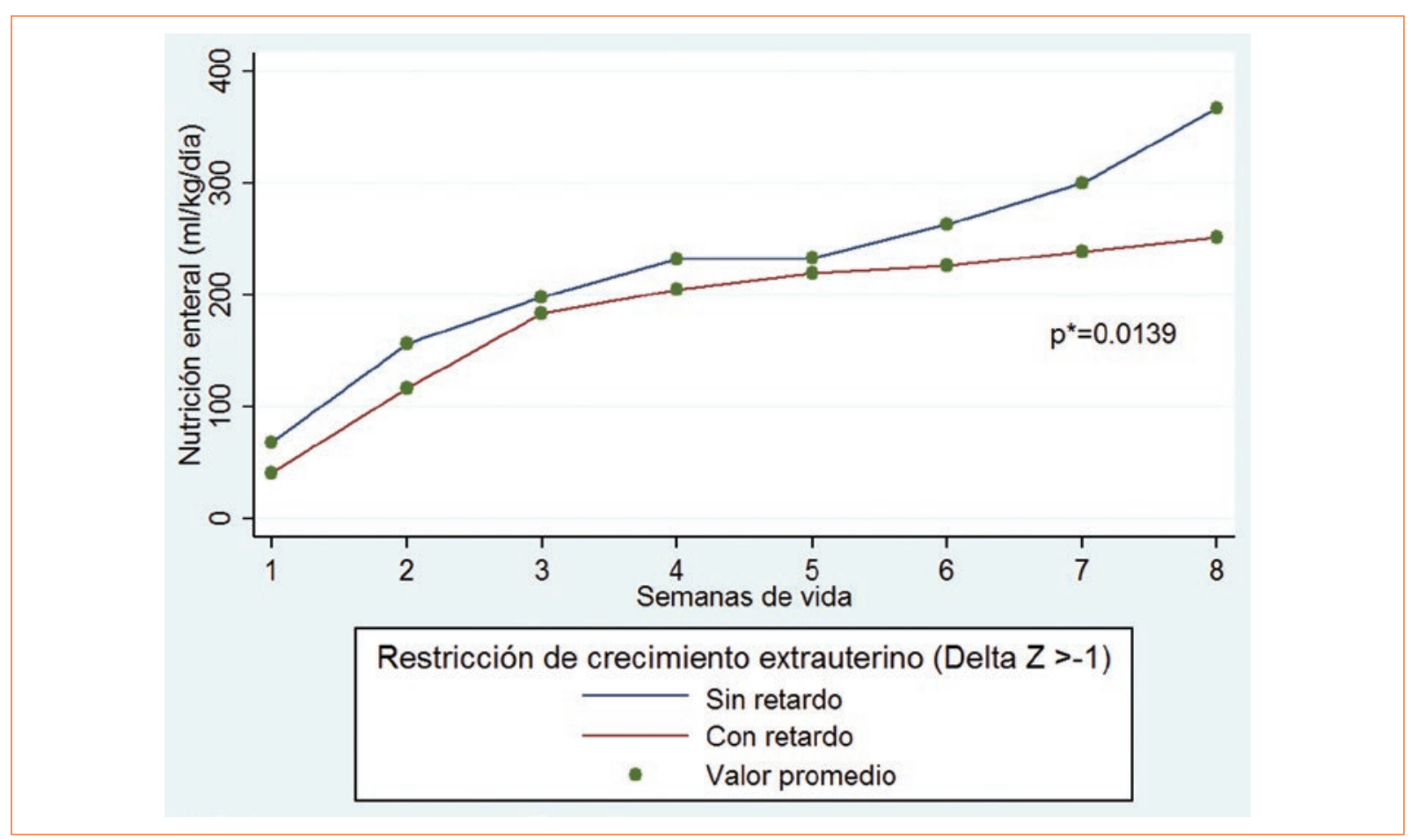

Figura 3. Comparación del promedio de nutrición enteral por semanas de vida según presencia de restricción de crecimiento extrauterino. Comparación por Anova de medidas repetidas de dos factores entre pacientes con y sin restricción extrauterina examinando el efecto de la nutrición enteral por semanas de vida. Se encontró una diferencia significativa entre los dos grupos $(\mathrm{p}<0.05)$. 


\section{Discusión}

El crecimiento extrauterino adecuado del recién nacido prematuro, especialmente en los pacientes de muy bajo peso al nacer, es uno de los objetivos de constante búsqueda en el cuidado neonatal, teniendo en cuenta que en ellos existen limitaciones en la rápida progresión enteral y, con ello, dificultades en alcanzar con prontitud la nutrición enteral plena, como lo encontramos en nuestro estudio, sumado a factores como la inmadurez de la función gastrointestinal, morbilidades, exposición a intervenciones médicas y farmacológicas que contribuyen a la malnutrición posnatal y al retardo en el crecimiento. De la misma forma lo evidencia Embleton, et al. en su estudio ${ }^{12}$, en el cual se concluye que la ingesta de nutrientes que satisfacen las recomendaciones dietéticas para el mantenimiento y crecimiento tardan en establecerse, y una vez establecidas, raramente se mantienen durante la estadía en el hospital en los recién nacidos prematuros, lo que conlleva a un déficit de nutrientes acumulativo ${ }^{12}$. Por otra parte, el estudio multicéntrico de Hobar, et al, en el que se evalúa el crecimiento de los recién nacidos de muy bajo peso al nacer durante la hospitalización y donde se excluyeron las principales morbilidades que han demostrado repercutir en el peso durante la estadía inicial en la UCIN, analizó 362,833 pacientes con un peso entre 501 y 1,500 gramos, sin defectos congénitos mayores al nacer, nacidos entre el año 2000 y el 2013, y se encontró un aumento en el promedio de velocidad de crecimiento de 11.8 a $12.9 \mathrm{~g} / \mathrm{kg}$ por día y una falla en el crecimiento posnatal que disminuyó de $64.5 \%$ a $50.3 \%$, lo que muestra que la falla grave de crecimiento posnatal pasó a ser del $39.8 \%$ al $27.5 \%$. Sin embargo, a pesar de la mejoría, en 2013, la mitad de los niños presentaban insuficiencia en el crecimiento posnatal y un cuarto (27.5\%) presentaban una insuficiencia grave en el crecimiento posnatal (menor al percentil 3) ${ }^{13}$. Los resultados de este estudio nos llevan a pensar que hay otros factores asociados para alcanzar las metas de crecimiento además de las comorbilidades.

Los objetivos nutricionales para los prematuros durante la hospitalización son una ingesta energética de $120 \mathrm{kcal} / \mathrm{kg} /$ día para suplir el gasto energético en reposo, actividad, termorregulación, alimentación, gasto fecal y el crecimiento, lo cual se alcanza con un aporte de $160 \mathrm{ml} / \mathrm{kg} / \mathrm{día}$ de fórmula para prematuros de $24 \mathrm{kcal} / \mathrm{oz}$ o leche materna fortificada, ajustando el volumen necesario y tolerado para garantizar un ganancia de peso de $15 \mathrm{a} 18 \mathrm{~g} / \mathrm{kg} / \mathrm{día}^{14}$. En los prematuros con muy bajo peso al nacer o muy prematuros, se recomienda el inicio temprano de la alimentación enteral trófica con calostro de la madre, dentro de las primeras 24 horas de vida, sumado a un esquema agresivo de nutrición parenteral, es decir, un aporte elevado de proteínas y lípidos y suficientes carbohidratos ${ }^{15}$. Esta conducta podría acortar el tiempo necesario para llegar al periodo de crecimiento estable. En nuestro estudio, el $42.1 \%$ de los recién nacidos en ambos grupos alcanzó la nutrición enteral plena en la segunda semana de vida. El aporte kilocalórico total al cabo de los días 56 a 62 fue de $234 \mathrm{kcal} / \mathrm{kg} / \mathrm{día}$, con volumen de nutrición enteral plena de $292.5 \mathrm{ml}$. Más de la mitad de los pacientes recibió nutrición parenteral, con una duración promedio de la misma de 12 días, y en la mayoría de los recién nacidos se inició NTP temprana, entre el primer y tercer día de vida $(90.9 \%)$, correspondiendo a las primeras 24 horas el $72.22 \%$.

En nuestro estudio planteamos posibles factores asociados al crecimiento posnatal adecuado en los recién nacidos prematuros de menos de 1,500 gramos al nacer que se hospitalizan en la UCIN del Hospital de San José de Bogotá DC. Nosotros encontramos que la variable PBEG disminuye la probabilidad de alcanzar una condición nutricional adecuada y es un factor de riesgo (OR: 7.3; IC 95\%: 2.86-19.05; $p=0.000$ ). Además evidenciamos una asociación directa entre nacer con peso adecuado para la EG y una adecuada condición nutricional, probablemente la razón del modelo multivariado es porque el principal determinante de la desnutrición está dado por la estancia de la unidad, que indirectamente puede estar asociado a nacer con bajo peso, a tener comorbilidades y a su potencial genético para alcanzar el crecimiento programado para su $\mathrm{EG}^{16}$.

La única forma que puede disminuir la frecuencia de desnutrición es aumentando los aportes calóricos con metas en medidas antropométricas y no en valores establecidos, teniendo en cuenta las limitaciones en implementar el volumen por algunas patologías, ya que no tenemos una escala de gravedad. Este estudio demuestra que el aporte calórico es lo más importante, y debe implementarse individualmente y principalmente enteral.

\section{Conclusiones}

El nacer prematuro y tener un PBEG es un factor que incide para no lograr alcanzar una condición nutricional ideal.

Los prematuros recién nacidos con bajo peso para la EG al nacer tienen un riesgo de no alcanzar una condición nutricional ideal 7.3 veces superior al de un recién nacido pretérmino con adecuado peso para la $E G$ al nacer. 
Los prematuros con PBEG y mayor estancia hospitalaria presentan un mayor riesgo de no alcanzar una condición nutricional ideal que aquellos con PAEG.

Existe la posibilidad de que la paridad, las gestaciones, el déficit de surfactante, la restricción de crecimiento intrauterino y el embarazo gemelar tengan una relación con no alcanzar una condición nutricional ideal, variables que deben ser motivo de futuros estudios.

\section{Agradecimientos}

A los servicios de Pediatría, Archivo y Estadística del Hospital de San José, y en especial al Dr. Pablo Vásquez, quien cumplió la función de asesor metodológico.

\section{Conflicto de intereses}

Los autores declaran que este artículo no presenta conflicto de intereses.

\section{Financiamiento}

La presente investigación no ha recibido ayudas específicas provenientes de agencias del sector público, sector comercial o entidades sin ánimo de lucro.

\section{Responsabilidades éticas}

Protección de personas y animales. Los autores declaran que los procedimientos seguidos se conformaron a las normas éticas del comité de experimentación humana responsable y de acuerdo con la Asociación Médica Mundial y la Declaración de Helsinki.

Confidencialidad de los datos. Los autores declaran que han seguido los protocolos de su centro de trabajo sobre la publicación de datos de pacientes.
Derecho a la privacidad y consentimiento informado. Los autores han obtenido el consentimiento informado de los pacientes y/o sujetos referidos en el artículo. Este documento obra en poder del autor de correspondencia.

\section{Bibliografía}

1. Hack M, Flannery DJ, Schluchter M, Cartar L, Borawski E, Klein N. Outcomes in young adulthood for very-low-birth-weight infants. N Engl $J$ Med. 2002;346(3):149-57.

2. Blencowe H, Cousens S, Oestergaard MZ, Chou D, Moller AB, Narwal R, et al. National, regional, and worldwide estimates of preterm birth rates in the year 2010 with time trends since 1990 for selected countries: a systematic analysis and implications. Lancet. 2012;379:2162-72.

3. Julie R. Gooding, MD, Richard E. McClead Jr, MD. Initial Assessment and Management of the Newborn. Pediatr Clin N Am. 2015;62:345-65

4. Lee PA, Chernausek SD, Hokken-Koelega AC, Czernichow P. International Small for Gestational Age Advisory Board 2003 International SmaII for Gestational Age Advisory Board consensus development conference statement: management of short children born small for gestational age, April 24-October 1, 2001. Pediatrics. 2003;111:1253-61.

5. Yu HJ, Kim ES, Kim JK, Yoo HS, Ahn SY, Chang YS, et al. Outcomes of small for gestational age micropremies depending on how young or how small they are. Korean J Pediatr. 2011;54(6):246-52.

6. Liu L, Johnson H, Cousens S, Perin J, Scott S, Lawn JE, et al.; Child Health Epidemiology Reference Group of WHO and UNICEF. Global, regional, and national causes of child mortality: an updated systematic analysis for 2010 with time trends since 2000. Lancet. 2012:379(9832):2151-61.

7. Zamorano C, Guzman J, Baptista H, Fernández L. Pérdida de peso corporal y velocidad de crecimiento postnatal en recién nacidos menores de 1,500 gramos durante su estancia en un hospital de tercer nivel de atención. Perinatol Reprod Hum. 2012;26(3):187-93.

8. Martin CR, Brown YF, Ehrenkranz RA, O'Shea TM, Allred EN, Belfort MB, et al. Nutritional practices and growth velocity in the first month of life. Pediatrics. 2009;124:649-57.

9. Guilfoy VM, Wright-Coltart S, Leitch CA, Denne SC. Energy expenditure in extremely low birth weight infants near time of hospital discharge. J Pediatr. 2008;153:612-5.

10. Griffin IJ. Nutritional assessment in preterm infants. Nestle Nutr Workshop Ser Pediatr Program. 2007;59:177-88.

11. Lubchenco LO, Hansman $C$, Boyd $E$. Intrauterine growth in length and head circumference as estimated from live births at gestational ages from 26 to 42 weeks. Pediatrics. 1966;37:403-8.

12. Embleton NE, Pang N, Cooke RJ. Postnatal malnutrition and growth retardation: an inevitable consequence of current recommendations in preterm infants? Pediatrics. 2001;107:270.

13. Horbar JD, Ehrenkranz RA, Badger GJ, Edwards EM, Morrow KA, Soll RF, et al. Weight Growth Velocity and Postnatal Growth Failure in Infants 501 to 1500 Grams: 2000-2013. Pediatrics. 2015;136:e84.

14. Griffun I. Growth management in preterm infants. En: UpToDate, Jan 16, 2017 (Último acceso: 9 de mayo de 2017).

15. Guía de práctica clínica del recién nacido prematuro. Nutrición del recién nacido prematuro. Ministerio de Salud y Protección Social. Colombia, Bogotá DC, 2013.

16. Prentice $P$, Acerini $C L$, Eleftheriou $A$, Hughes IA, Ong KK, Dunger DB Cohort Profile: the Cambridge Baby Growth Study (CBGS). Int J Epidemiol. 2016;45(1):35.a-g. 